\title{
LA ESTIMACIÓN DE LA CAPACIDAD EMPRENDEDORA EN Países de aMÉ́rica LatiNa Y EL CARIBE
}

\author{
THE ESTIMATION OF ENTREPRENEURIAL CAPACITY IN \\ COUNTRIES OF LATIN AMERICA AND THE CARIBBEAN
}

\author{
Teodoro Reyes Fong, ${ }^{* 1}$ José Luis Esparza Aguilar, ${ }^{2}$ Martín Álvarez Ochoa ${ }^{1}$ Sergio Felipe López \\ Jiménez, ${ }^{1}$ \\ 1 Universidad de Colima; teodoro_reyesf@ucol.mx; alvarezochoa@ucol.mx; sflopezj@ucol.mx. \\ 2 Universidad de Quintana Roo; jlesparza@uqroo.edu.mx
}

RESUMEN

ABSTRACT

Este trabajo estima la capacidad emprendedora de algunos países de América Latina y el Caribe a partir de la obtención de sus índices de productividad, para conocer cuáles son los factores determinantes en el emprendimiento. Se estimaron los Índices de Productividad de Malmquist, y para ello, se utilizó el Análisis Envolvente de Datos con rendimientos variables y orientación output; las variables empleadas fueron: la intención emprendedora, la tasa de actividad emprendedora, las oportunidades percibidas, las capacidades percibidas y la tasa de miedo al fracaso; las cuales se obtuvieron de la información del Clobal Entrepreneurship Monitor. Los resultados revelan que la intención y la actividad emprendedora depende principalmente de las oportunidades y las capacidades percibidas, así como de la tasa de miedo al fracaso; por lo que, se evidencia que los países que tienen una mayor capacidad emprendedora, desarrollan mayores acciones de innovación, y por lo tanto obtienen una mayor ventaja competitiva.

PALABRAS CLAVE: Innovación; Emprendimiento; Actitudes Emprendedoras; Índice de Malmquist.
This work estimates the entrepreneurial capacity of some countries in Latin America and the Caribbean based on obtaining their productivity indexes, in order to know what are the determining factors in entrepreneurship. The Malmquist Productivity Indices were estimated, and for this, the Data Envelopment Analysis with variable returns and output orientation was used, the variables used were: the entrepreneurial intentions, total early-stage entrepreneurial activity, perceived opportunities, perceived capabilities and the fear of failure rate; which were obtained from the Clobal Entrepreneurship Monitor information. The results reveal that the intention and the entrepreneurial activity depends mainly on the opportunities and the perceived capabilities, as well as on the fear of failure rate; therefore, it is evident that the countries that have a greater entrepreneurial capacity, develop greater innovation actions, and therefore obtain a greater competitive advantage.

KEYWORDS: innovation; entrepreneurship; entrepreneurial attitudes; malmquist index. 


\section{Introducción}

En América Latina y en el mundo se ha dado prioridad a las acciones de emprendimiento, ya que son las empresas el motor que impulsa el crecimiento y la competitividad en el desarrollo de las economías y coadyuvan en el bienestar de la sociedad en general; algunos autores consideran que el crecimiento de las economías depende de las capacidades de innovación y emprendimiento (Mendez, 2002; Scheel, 2012). En este sentido, la capacidad emprendedora es vital para conseguir ventaja competitiva de una región frente a otras (Porter, 1991)

La importancia que juega la capacidad emprendedora en la creación de nuevas empresas se destaca en algunos estudios (MacMillan, Siegel, \& Narasimha, 1985; Khan, 1986; Sandberg \& Hofer, 1987), y es que el emprendedor es un agente de cambio en la región en la cual se encuentre (Qian \& Acs, 2013; Sanchez, Garcia, \& Mendoza, 2015).

La información disponible sobre la capacidad emprendedora es limitada por lo que se utilizaron los datos del proyecto Clobal Entrepreneurship Monitor (GEM), el cual deriva de una investigación conjunta entre el Babson College (Estados Unidos) y la London Business School (Reino Unido), actualmente es el mayor proyecto sobre la investigación del emprendimiento, y el propósito fundamental ha sido analizar la relación entre la actividad emprendedora y el crecimiento de las economías.

El objetivo de este estudio es doble, por una parte, conocer cuál es la capacidad emprendedora en algunos países de América Latina y el Caribe, y por otra, mostrar un panorama general sobre la evolución de la investigación en emprendimiento con el uso de los datos del proyecto GEM.

El trabajo se estructura en cinco apartados: el primero corresponde a la revisión de los estudios empíricos previos; el segundo apartado presenta el enfoque metodológico aplicado, la elección de la muestra y la justificación de las variables; en el tercer apartado se presentan los resultados; en el cuarto apartado se discuten los resultados, y en el último, se presentan las principales conclusiones derivadas del estudio.

\section{Actitudes Emprendedoras}

Hoy en día como parte de las políticas públicas en la mayoría de los países se ha fomentado el emprendimiento, ya que es considerado como una fuente de crecimiento y competitividad de las economías y por lo tanto del bienestar social. Por ello, es importante hacer una diferenciación de la capacidad emprendedora a partir de los sistemas regionales en función de los resultados que derivan de los mismos (Sanchez, Garcia, \& Mendoza, 2015).

La información proporcionada por el GEM es muy importante ya que a partir de ella es posible conocer la realidad de la actividad de emprendimiento de diversos países e incluso se pueden realizar estudios comparativos entre regiones. El proyecto del GEM inicia en $1997 \mathrm{y}$ a la fecha tiene una base de datos de 22 años, más de 200,00o encuestas aplicadas por año, datos de análisis de más de 100 economías, y para ello, hay una participación de más de 500 investigadores especialistas en temas de emprendimiento, más de 300 académicos e investigadores de diversas instituciones y más de 200 instituciones de financiamiento (Global Entrepreneurship Monitor, 2019). Cada día son más los trabajos que han hecho uso de la información del GEM, Ramos, Martinez, Medina, \& Ruiz (2013) identifican 86 artículos publicados en revistas del Social Science Citation Index (SSCI), los cuales han utilizado los datos del GEM.

A pesar de que el inicio del GEM es reciente, existe una diversidad de trabajos que utilizan la información derivada del proyecto para realizar estudios en el campo del emprendimiento, en la tabla 1 se muestran 2 áreas principales en las cuales se han realizado diversas investigaciones.

Tabla 1 Estudios con datos derivados del CEM

Tema de investigación

Análisis de los factores determinantes de la actividad emprendedora.

Comparación de la actividad emprendedora entre regiones o países.
Trabajos

Galindo, Mendez, \& Alfaro (2010), Pete, y otros (2011), Castaño (2012), Klyver \& Schenkel (2013), Stuetzer, Obschonka, Brixy, Sternberg, \& Cantner (2014), Henry, Foss, Fayolle, Walker, \& Duffy (2015), Tsai, Chang, \& Peng (2016), Mickiewicz, Wedzerai, Theodorakopoulos, \& Hart (2017), Elbahlawan (2018), Apetrei, Sanchez, \& Sapena (2019), Lafuente, Acs, Sanders, \& Szerb (2019).

Alvarez, Valencia, Barraza, \& Legato (2010), Merino \& Vargas (2011), Sternberg (2012), Stam (2013), Kim \& Li (2014), Diaz, Diaz, Sanchez, \& Hernandez (2015), Woodside, Mir, \& Coduras (2016), Beynon, Jones, \& Pickernell (2016), Lecuna, Cohen, \& Chavez (2017), Farinha, Ferreira, \& Nunes (2018), Dilli \& Westerhuis (2018), Chuang, Yeh, \& Lin (2019). 
A continuación se describen las variables con las que se estima la percepción y la actitud emprendedora: Oportunidades percibidas, capacidades percibidas, miedo al fracaso, intención emprendedora y tasa de emprendimiento temprano; en este estudio se utilizan las definiciones recogidas en el proyecto GEM.

\section{Oportunidades Percibidas}

Una característica fundamental en el tema de emprendimiento es la que se relaciona con las oportunidades percibidas, debido a que pocas o malas oportunidades en los negocios repercuten en una inadecuada actividad emprendedora (Krueger, 2000). Las oportunidades percibidas son la percepción que las personas tienen sobre las circunstancias para iniciar un negocio (Amoros, 2011); sin embargo, las oportunidades no necesariamente implican la creación de nuevas empresas, debido a que se pueden presentar en diferentes momentos y en diversas formas (Henley, 2007). Esta variable ha sido utilizada en los estudios de Krueger, Reilly, \& Carsrud (2000), Arenius \& Minniti (2005), Acs (2006) y, Beynon, Jones, \& Pickernell (2016).

\section{Capacidades Percibidas}

Algunos autores han identificado las capacidades que deben tener los emprendedores efectivos (McGee, Peterson, Mueller, \& Sequeira, 2009); para Acs, Desai, \& Hessels (2008) las percepciones de la gente sobre su entorno y sobre ellos mismos los acercan o alejan del emprendimiento. En este estudio, las capacidades percibidas están representadas por el porcentaje que representan las personas emprendedoras quienes creen que poseen las competencias como habilidades, conocimientos y experiencias necesarias para iniciar un negocio (Beynon, Jones, \& Pickernell, 2016). Algunos autores que han empleado esta variable son: Acs, Desai, \& Hessels (2008), McGee, Peterson, Mueller, \& Sequeira (2009), Beynon, Jones, \& Pickernell (2016), y Soria, Zuñiga, \& Ruiz (2016).

\section{Miedo Al Fracaso}

El temor al emprendimiento limita la creación de empresas, ya que algunas personas tienen temor al riesgo (Arenius \& Minniti, 2005; Vaillant \& Lafuente, 2007). En el caso de los empresarios de los Estados Unidos de Norteamérica, se acepta el fracaso en las empresas ya que consideran que ello es una experiencia positiva porque les ayuda a mejorar en el conocimiento y la competencia empresarial.

Algunos estudios en esta línea muestran los siguientes hallazgos: en el caso de China y Pakistán, Ul Haq, Usman, Hussain, \& Anjam (2014) no encontraron una relación entre el miedo al fracaso y el espíritu emprendedor.
No obstante, Bosma \& Kelley (2018) demuestran que en países basados en el manejo eficiente de sus factores, los empresarios con mayor miedo al fracaso son los que muestran menores intenciones emprendedoras; en el caso de Tailandia, Suecia, Chile y Arabia Saudita, se identifican mejores oportunidades a pesar de las mayores tasas de miedo al fracaso. Los estudios que han utilizado esta variable son: Arenius \& Minniti (2005), Vaillant \& Lafuente (2007), Ul Haq, Usman, Hussain, \& Anjam (2014), y Bosma \& Levie (2019).

\section{Intención Emprendedora}

La intención emprendedora puede ser de tres tipos: personal, social o cultural, y mide la tendencia que tienen las economías hacia el emprendimiento, la cual puede ser motivada por la necesidad o la oportunidad (Beynon, Jones, \& Pickernell, 2016). En consecuencia, la intención emprendedora es el actuar o las intenciones que se tienen para emprender un negocio (Bosma \& Kelley, 2018); la definición de la intención emprendedora que considera el GEM es con relación a las personas que tienen la intención de iniciar un negocio en los próximos tres años. Esta variable ha sido utilizada en los estudios de Autio, Keeley, Klofsten, Parker, \& Hay (2001), Ruiz \& Garcia (2013), y Beynon, Jones, \& Pickernell (2016).

\section{Tasa de Emprendimiento Temprano}

La tasa de emprendimiento temprano de acuerdo a lo definido por el GEM, es el porcentaje de personas encuestadas entre 18 y 64 años que están en proceso de abrir un negocio o aquellas que tienen un negocio de hasta 3.5 años de antigüedad. En este sentido, es importante tener en consideración que las decisiones para emprender pueden depender de algunos factores como la motivación personal (Park, Kim, \& Lee, 1999; Hadjimanolis, 2000; Levenburg, Magal, \& Kosalge, 2006) que puede estar vinculada a las condiciones del entorno (Sanchez, Garcia, \& Mendoza, 2015), y por lo tanto, poner en marcha mayores acciones de innovación (Feeny \& Rogers, 2003; Chang \& Ahn, 2005; Thornhill, 2006; Jimenez \& Sanz, 2006).

Por otra parte, las características particulares de cada país (Acs \& Audretsch, 1987; Acs \& Audretsch, 1988; Dijk, Den, Menkveld, \& Thurik, 1997; Frenkel, Shefer, Koschatzky, \& Walter, 2001; Noronha, Cesario, \& Fernandes, 2006) pueden incidir en mayores o menores necesidades sociales y por lo tanto, en la tasa de emprendimiento temprano (Vidal, 2008). Algunos trabajos que han utilizado esta variable son los de Beynon, Jones, \& Pickernell (2016), Zamora (2018), y Diaz, Hernandez, Sanchez, \& Postigo (2010). 


\section{Enfoque Teórico en Emprendimiento}

Existen diversos enfoques teóricos que se relacionan con el emprendimiento, sin embargo, son dos las que se enmarcan mejor a la aportación de este estudio: la teoría del empresario y la teoría de la eficiencia-X.

La teoría del empresario considera que la intención emprendedora depende de un estado de alerta a las oportunidades; por lo que el emprendedor exitoso, es aquel que aprovecha las oportunidades que otras personas no ven, o que percibe oportunidades en situaciones donde otras personas han llevado acciones emprendedoras asignando ciertos recursos en situaciones contingentes o de desequilibrio (Kirzner, 1979).

Ante la falta de estrategias y la ineficiencia de las organizaciones se crean oportunidades excelentes para los emprendedores; los emprendedores que tienen una visión clara también suelen ser más eficientes en sus procesos emprendedores, y por lo tanto son un empuje para el resto de empresas (Casson, 1982). En este contexto, la teoría de la eficiencia-X de Leibenstein (1966) analiza el papel que desempeñan los emprendedores, y considera que la ineficiencia se debe al mal aprovechamiento de los recursos en función de las oportunidades, por lo que los éxitos o fracasos estarán dados, por el buen o mal aprovechamiento por parte de los emprendedores.

Al considerar los procesos internos que se desarrollan para la actividad emprendedora, es importante el uso eficiente de los recursos, pero también las acciones para lograr una mayor competitividad en las organizaciones; es así como la actividad emprendedora resulta ser un impulsor para la innovación de las empresas, lo que impacta de manera positiva en el bienestar y en el crecimiento de las economías (Wennekers \& Thurik, 1999; Audretsch \& Fritsch, 2003; Carree \& Thurik, 2003). Con base en la literatura revisada, se plantea la siguiente hipótesis:

H1: La actividad emprendedora de las organizaciones tiene una correlación positiva con las acciones de innovación.

\section{Metodología}

De la lista de países de Latinoamérica y el Caribe que despliega en su estudio el proyecto GEM, se encontraron algunos que no presentan información consistente en el periodo de 2010 a 2017, los cuales fueron eliminados del estudio y por lo tanto, sólo se estimó la productividad de diez países de Latinoamérica y el Caribe durante dicho periodo (ver tabla 2). Para tal efecto, se empleó el índice de productividad total de Malmquist (IPM) el cual se fundamenta en la estimación de las funciones de distancia; con el objetivo de identificar las contribuciones del cambio en eficiencia y cambio tecnológico en la productividad, Fare et al. (1992) proponen la descomposición del Indice de Productividad Total de Malmquist.

La estimación de las funciones de distancia del IPM, se realizó utilizando el método de programación lineal DEA con una orientación output y con rendimientos variables. El DEA fue desarrollado por Charnes, Cooper y Rhodes (1978); el DEA es una técnica de programación matemática que estima una frontera envolvente empírica a partir de un conjunto de inputs y outputs del grupo de unidades analizadas (Coll \& Blasco, 2000). El DEA estima la eficiencia técnica de los procesos productivos a partir de un conjunto de recursos técnicos o procedimientos basándose en las cantidades en términos de outputs e inputs. Algunos estudios han utilizado esta herramienta para estudiar en efecto de las oportunidades en el emprendimiento (Anokhin, Wincent, \& Autio, 2011) y para conocer el efecto de la actividad emprendedora en la eficiencia de los países (Tasnim \& Afzal, 2018).

Para realizar las estimaciones, Fare et al. (1994), proponen estimar cuatro funciones de distancia que permitan medir el cambio del IPM en dos períodos. El modelo con orientación output se describe de la siguiente manera:

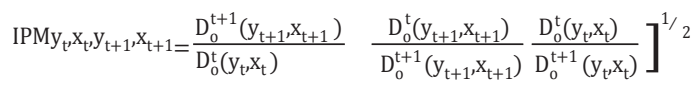

En el modelo matemático anterior, el cociente que está fuera de los corchetes mide el cambio en la eficiencia entre los períodos $t$ y $t+1$, siendo equivalente al cociente de la eficiencia técnica de Farrell en el período t+1 y en el período t; muestra los cambios en el tiempo o efecto catching-up de la eficiencia, dejando ver si la unidad evaluada se está acercando o alejando de la frontera eficiente. Este cambio en el nivel de eficiencia es consecuencia de la capacidad que tienen las organizaciones de incorporar progreso técnico en la gestión de su proceso productivo. La parte restante del modelo es la medida del cambio tecnológico, representado por la media geométrica de la transformación tecnológica entre los períodos t y $t+1$.

Si una unidad muestra entre dos períodos $t$ y $t+1$ un aumento en su productividad, el IPM tomará un valor mayor a 1; si por el contrario, la unidad pierde productividad a lo largo del período, el IPM tendrá un valor inferior a 1. En cambio, si no se produce variación en la productividad el valor del IPM será 1 . Sin embargo, es importante considerar que los componentes del IPM pueden tomar direcciones opuestas, por lo que cabe la posibilidad que mientras que uno aumenta el otro disminuya, y viceversa.

Por efecto catching-up se entiende el cambio en eficiencia propia de las organizaciones con 
respecto a la frontera de posibilidades productivas, es decir, la mejor forma de realizar sus procesos de gestión para alcanzar sus metas; y aparte se debe considerar el cambio técnico que indica las mejoras en innovación o la capacidad que tienen las organizaciones para implementar nuevas técnicas o procedimientos para alcanzar mejores niveles de competitividad.

Para realizar las estimaciones se utilizó el programa Data Envelopment Analysis desarrollado por Tim Coelli en el Centro para el análisis de la eficiencia y la productividad del Departamento de econometría de la Universidad de Nueva Inglaterra.

También se calculó la correlación entre las variables, para ello, se estimaron los coeficientes de correlación de Pearson, con el fin de conocer si las variables utilizadas es- tán o no linealmente correlacionadas entre sí. La estimación se realizó con el Statistical Package for the Social Science (SPSS).

\section{Resultados}

En la tabla 2 se agrupan las variables para la investigación; las variables input sobre la capacidad emprendedora utilizadas en este estudio fueron: las oportunidades percibidas, las capacidades percibidas y la tasa de miedo al fracaso.

Las variables output elegidas para estimar el factor total de productividad en emprendimiento fueron: la intención emprendedora y la tasa de actividad emprendedora; los datos se obtuvieron de la información del Global Entrepreneurship Monitor.

Tabla 2 Variables utilizadas en el análisis

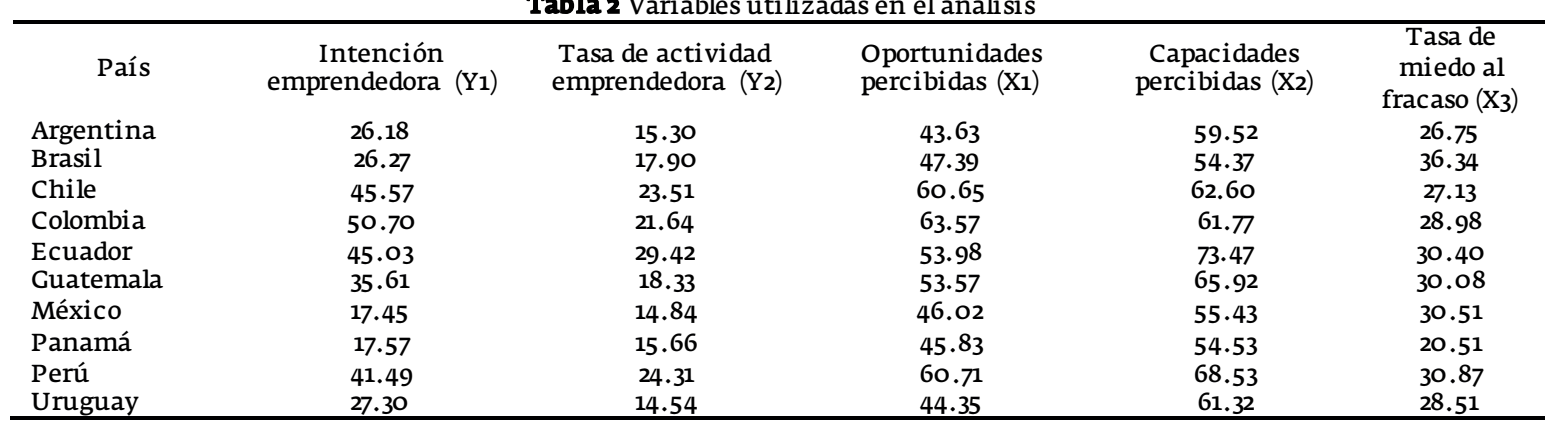

La información de la tabla 2 muestra los valores promedio de las variables seleccionadas para el estudio, con estos datos se estimaron los índices de productividad de Malmquist, por periodo y por país. El índice de productividad de Malmquist (tfpch) se integra por la forma adecuada de realizar la gestión de los procesos (effch) y por la capacidad de innovar o realizar cambios en las técnicas (techch).
La capacidad de gestionar los procesos (effch) se desagrega en la capacidad que se tiene de maximizar la combinación de los factores de que se dispone (pech) y por la forma de realizar cambios en la escala de operaciones (sech). Las estimaciones de la evolución del factor total de productividad por año se presentan en la tabla 3 .

Tabla 3 Factor total de productividad por año

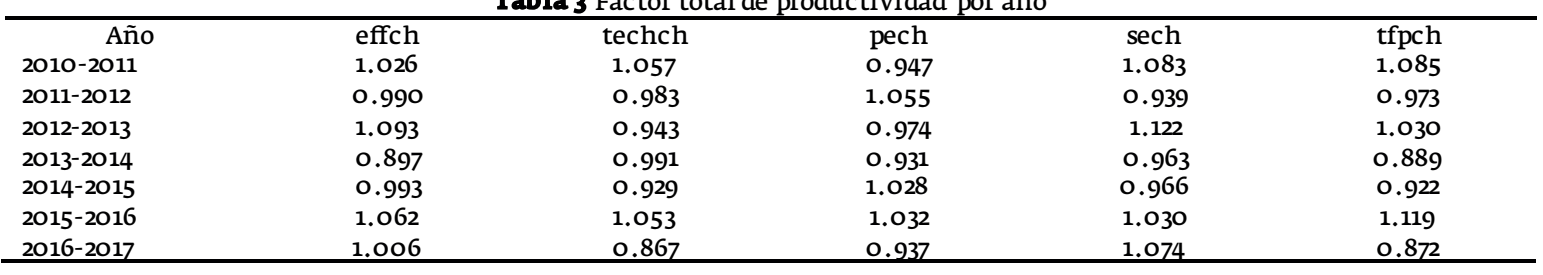

En las estimaciones del índice de productividad de Malmquist se puede ver durante el periodo 2010 a 2017 un incremento en el factor total de productividad en promedio (tfpch) para los años 2010-2011, 2012-2013 y 2015-2016, lo cual representa en promedio un incremento del $7.8 \%$. Este indicador se compone por el cambio tecnológico (techch) y el cambio en eficiencia (effch); el incremento se explica principalmente por el promedio del cambio en eficiencia que presenta un $6 \%$.
El cambio en eficiencia se desagrega en cambio en eficiencia pura (pech) y cambio en eficiencia a escala (sech); por lo que conforme a los resultados presentados, la eficiencia se debe en mayor medida al cambio en eficiencia a escala, el cual muestra un incremento del 7.8\%.

La tabla 4 muestra la relación de países con los resultados del factor total de productividad y su desagregación. 
Tabla 4. Factor total de productividad y su desagregación por país

\begin{tabular}{llllll}
\hline \multicolumn{1}{c}{ País } & effch & techch & pech & sech & tfpch \\
Argentina & 1.078 & 0.954 & 0.966 & 1.116 & 1.029 \\
Brasil & 1.002 & 0.979 & 1.000 & 1.002 & 0.981 \\
Chile & 1.000 & 0.973 & 1.000 & 1.000 & 0.973 \\
Colombia & 1.000 & 0.977 & 1.000 & 1.000 & 0.977 \\
Ecuador & 1.000 & 0.979 & 1.000 & 1.000 & 0.979 \\
Cuatemala & 0.980 & 0.982 & 0.982 & 0.998 & 0.962 \\
México & 0.967 & 0.988 & 0.934 & 1.035 & 0.956 \\
Panamá & 1.052 & 0.988 & 1.000 & 1.052 & 1.039 \\
Perú & 1.011 & 0.964 & 1.011 & 1.000 & 0.975 \\
Uruguay & 0.995 & 0.943 & 0.963 & 1.033 & 0.938 \\
$\quad$ Promedio & 1.008 & 0.973 & 0.985 & 1.023 & 0.980 \\
\hline
\end{tabular}

Los países de América Latina y el Caribe que fueron incluidos en el estudio (tabla 4), presentan un factor total de productividad en promedio de 0.980 , lo cual representa un decremento del $2 \%$ para el total de los países analizados. Este decremento se debe en mayor medida al cambio tecnológico que tiene en promedio un decremento del $2.7 \%$, puesto que el cambio en eficiencia muestra un incremento muy bajo que representa el $0.8 \%$.
El cambio en eficiencia se explica por el incremento promedio del $2.3 \%$ del cambio en eficiencia a escala, ya que el cambio en eficiencia pura tuvo un decremento promedio del $1.5 \%$.

Finalmente en la tabla 5 , se muestran las estimaciones de los índices de correlación de Pearson para cada una de las variables empleadas en el estudio.

Tabla 5 Índices de correlación de Pearson entre variables

\begin{tabular}{|c|c|c|c|c|c|}
\hline & $\begin{array}{c}\text { Intención } \\
\text { emprendedora }\end{array}$ & $\begin{array}{c}\text { Tasa de } \\
\text { actividad } \\
\text { emprendedora }\end{array}$ & $\begin{array}{l}\text { Oportunidades } \\
\text { percibidas }\end{array}$ & $\begin{array}{l}\text { Capacidades } \\
\text { percibidas }\end{array}$ & $\begin{array}{c}\text { Tasa de miedo al } \\
\text { fracaso }\end{array}$ \\
\hline \multirow[t]{2}{*}{ Intención emprendedora } & 1 & $.823^{* *}$ & $.900^{* *}$ & $.735^{* *}$ & .204 \\
\hline & & .003 & .000 & .015 & .573 \\
\hline \multirow{2}{*}{$\begin{array}{l}\text { Tasa de actividad } \\
\text { emprendedora }\end{array}$} & $.823^{* *}$ & 1 & $.739^{*}$ & $.809^{* *}$ & .221 \\
\hline & .003 & & .015 & .005 & .539 \\
\hline \multirow[t]{2}{*}{ Oportunidades percibidas } & $.900^{* *}$ & $.739^{*}$ & 1 & .551 & .134 \\
\hline & .000 & .015 & & .099 & .712 \\
\hline \multirow[t]{2}{*}{ Capacidades percibidas } & $.735^{*}$ & $.809^{* *}$ & .551 & 1 & .155 \\
\hline & .015 & .005 & .099 & & .670 \\
\hline \multirow[t]{2}{*}{ Tasa de miedo al fracaso } & .204 & .221 & .134 & .155 & 1 \\
\hline & .573 & .539 & .712 & .670 & \\
\hline
\end{tabular}

Nota. ${ }^{* *} \mathrm{p}<0,01 ;{ }^{*} \mathrm{p}<0,05$.

Los coeficientes de correlación lineal de Pearson muestran evidencia de una alta correlación lineal positiva entre las oportunidades percibidas y las capacidades percibidas, con relación a la intención emprendedora y la tasa de actividad emprendedora; no obstante, en el caso de la tasa de miedo al fracaso no se logra encontrar correlaciones significativas con la intención emprendedora y la tasa de actividad emprendedora.
Discusión

En la tabla 6 se presenta una agrupación por países con los indicadores más altos y más bajos en los índices de productividad y eficiencia, así como su desagregación en los factores que componen a cada uno de esos índices.

Tabla 6 Países agrupados por los índices más altos y más bajos

\begin{tabular}{|c|c|c|c|c|c|}
\hline & tfpch & techch & effch & pech & sech \\
\hline Indicadores bajos & $\begin{array}{l}\text { Uruguay, México } \\
\text { y Guatemala }\end{array}$ & $\begin{array}{l}\text { Uruguay, } \\
\text { Argentina y Perú }\end{array}$ & $\begin{array}{l}\text { México y } \\
\text { Guatemala }\end{array}$ & $\begin{array}{l}\text { México, } \\
\text { Uruguay y } \\
\text { Argentina }\end{array}$ & Guatemala \\
\hline
\end{tabular}


Del primer grupo de países, Panamá, Argentina, Brasil y Ecuador, los cuales presentan mejores indicadores en la productividad considerando la intención emprendedora y la tasa de actividad emprendedora; Panamá tiende a la aplicación de técnicas de emprendimiento más innovadoras (techch) (Feeny \& Rogers, 2003; Chang \& Ahn, 2005; Thornhill, 2006; Jimenez \& Sanz, 2006), mientras que Argentina, Ecuador y Brasil buscan las mejoras sólo por la capacidad de gestión (effch) pero no implementan procesos innovadores (Park, Kim, \& Lee, 1999; Hadjimanolis, 2000; Levenburg, Magal, \& Kosalge, 2006). Al momento de analizar los factores determinantes de la capacidad de gestión en sus procesos de emprendimiento de este grupo de países, Panamá, Argentina y Brasil han desarrollado sus actividades de emprendimiento con relación a un tamaño óptimo (sech) (Acs \& Audretsch, 1987; Acs \& Audretsch, 1988; Dijk, Den, Menkveld, \& Thurik, 1997; Frenkel, Shefer, Koschatzky, \& Walter, 2001; Noronha, Cesario, \& Fernandes, 2006), es decir, han combinado de mejor manera su percepción de oportunidades, la percepción de capacidades y la tasa de miedo al fracaso.

En el segundo grupo de países, Uruguay, México y Guatemala son los que presentan los indicadores más bajos; los resultados muestran que Uruguay es el país que aplica técnicas más innovadoras en el emprendimiento (techch) (Feeny \& Rogers, 2003; Chang \& Ahn, 2005; Thornhill, 2006; Jimenez \& Sanz, 2006), en cambio, México y Guatemala son los países que presentan buenas técnicas de emprendimiento (Park, Kim, \& Lee, 1999; Hadjimanolis, 2000; Levenburg, Magal, \& Kosalge, 2006) pero no de forma innovadora (effch). En este grupo, Uruguay y México en su actividad emprendedora no han utilizado correctamente la percepción de oportunidades, la percepción de capacidades y la tasa de miedo al fracaso (pech) (Park, Kim, \& Lee, 1999; Hadjimanolis, 200o; Levenburg, Magal, \& Kosalge, 2006); no así Cuatemala, el cual intenta desarrollar actividades de emprendimiento a una escala óptima (sech) (Acs \& Audretsch, 1987; Acs \& Audretsch, 1988; Dijk, Den, Menkveld, \& Thurik, 1997; Frenkel, Shefer, Koschatzky, \& Walter, 2001; Noronha, Cesario, \& Fernandes, 2006), sin embargo, sus resultados en emprendimiento finalmente resultan con valores bajos.

En función a los resultados de los índices de correlación de Pearson mostrados en la tabla 5 , se encontró que cuando se perciben oportunidades y capacidad de emprendimiento, hay una mayor intención y actividad emprendedora, es decir, una vertiente se da por la voluntad de iniciar un negocio, la cual puede ser motivada por la necesidad o la oportunidad (Park, Kim, \& Lee, 1999; Hadjimanolis, 2000; Feeny \& Rogers, 2003; Chang \& Ahn, 2005; Jimenez \& Sanz, 2006; Levenburg, Ma- gal, \& Kosalge, 2006; Thornhill, 2006; Beynon, Jones, \& Pickernell, 2016), y la otra, por la iniciativa que se tiene de emprender un negocio, pero siempre caracterizados por las condiciones de entorno de cada país (Acs \& Audretsch, 1987; Acs \& Audretsch, 1988; Dijk, Den, Menkveld, \& Thurik, 1997; Frenkel, Shefer, Koschatzky, \& Walter, 2001; Noronha, Cesario, \& Fernandes, 2006). Por otra parte, el temor al fracaso podría ser una limitante para la creación de empresas (Cacciotti, Hayton, Mitchell, \& Giazitzoglu, 2016; Kollmann, Stockmann, \& Kensbock, 2017), ya que algunas personas podrían tener cierta inquietud por el riesgo que representa el emprendimiento.

Los hallazgos coinciden con algunos autores, ya que por una parte, muestran que las actividades de emprendimiento presentan una mayor sensibilidad a la combinación de los factores sobre la percepción de oportunidades, la percepción de capacidades y la tasa de miedo al fracaso (Beynon, Jones, \& Pickernell, 2016); y por otra, se evidencia una relación positiva entre la innovación y las actividades emprendedoras (Anokhin, Wincent, \& Autio, 2011; Sanchez, Garcia, \& Mendoza, 2015; Tasnim \& Afzal, 2018). De acuerdo con lo anteriormente expuesto, los resultados obtenidos muestran evidencias significativas de que la actividad emprendedora tiene una correlación positiva con las acciones de innovación, por lo que la hipótesis H1 no se rechaza.

\section{Conclusiones}

En este estudio se han analizado las capacidades emprendedoras en algunos países de América Latina y el Caribe, para ello se ha utilizado el Análisis Envolvente de Datos, el cual es una herramienta de estimación no paramétrica ampliamente aplicado en estudios de análisis de eficiencia y productividad, que permite conocer el nivel de desempeño de cada país, y que mediante la estimación del Índice de Productividad de Malmquist permite conocer cuáles son los factores determinantes y significativos en la capacidad emprendedora.

La relación entre la innovación y el emprendimiento es un factor determinante en los países desarrollados, ya que estos presentan un mejor crecimiento económico y mayor bienestar social, ya que la alta innovación que les caracteriza coadyuva a una mayor actividad emprendedora. Estos países presentan una mejor capacidad emprendedora con relación a la percepción de oportunidades, percepción de capacidades y la tasa de miedo al fracaso, lo cual posiblemente sea resultado de la capacidad que tienen las empresas para hacer frente a los diversos factores de entorno, y así alcanzar mejores ventajas competitivas.

En los países de economías emergentes como es el caso de este estudio, la actividad 
emprendedora presenta una notable desventaja competitiva, lo cual deriva posiblemente de una preocupación mayor en realizar procesos eficientes que les ayuden a reducir costos y a posicionarse como organizaciones más productivas; es decir, este grupo de países presentan limitaciones en su capacidad innovadora, motivado quizás porque no tienen un adecuado reconocimiento de las oportunidades, la percepción de sus capacidades es limitada y existe un alto nivel de miedo al fracaso. En contraparte, se enfocan solamente a buscar la utilización adecuada de sus recursos para poder hacer frente a los factores de entorno y así salvar el déficit que tienen en prácticas de gestión innovadoras, lo cual no les permite alcanzar una ventaja competitiva mediante las economías de escala.

Cuando las personas perciben que tienen oportunidades para los negocios, muestran mayores intenciones para el emprendimiento; en cambio, si la percepción resulta sobre las capacidades de las personas para realizar algún negocio, entonces las intenciones se materializan en actividades emprendedoras, es decir, pasan de la intención emprendedora a la acción de realizar algún negocio.

La evolución de la investigación en temas de emprendimiento ha tenido un crecimiento acelerado, y se han llevado a cabo una gran cantidad de estudios, los cuales se orientan con la línea del emprendimiento, y cuya base para su desarrollo han sido los datos generados por el Global Entrepreneurship Monitor. Este estudio podría ser de utilidad para el avance de trabajos futuros que pretendan identificar los factores determinantes de los niveles de productividad en las acciones emprendedoras de las regiones o los países.

\section{Referencias}

Acs, Z. (2006). How Is Entrepreneurship Good for Economic Growth? Innovations: Technology, Governance, Globalization, 1(1), 97-107.

Acs, Z., \& Audretsch, D. (1987). Innovation, Market Structure, and Firm Size. Review of Economics and Statistics, 69(4), 567-574.

Acs, Z., \& Audretsch, D. (1988). Innovation in Large and Small Firms: An Empirical Analysis. American Economic Review, 78, 678-690.

Acs, Z., Desai, S., \& Hessels, J. (2008). Entrepreneurship, economic development and institutions. Small Business Economics, 31(3), 219-234.

Alvarez, A., Valencia, P., Barraza, S., \& Legato, A (2010). Factors determining the entrepreneurial consolidation in Latin America. African Journal of Business Management, 4(9), 1717-1722. Amoros, J. (2011). El proyecto Global Entrepreneurship Monitor (GEM): Una aproximación desdeel contextolatinoamericano. Revista Latinoamericana de Administración(46), 1-15.

Anokhin, S., Wincent, J., \& Autio, E. (2011). Operationalizing opportunities in entrepreneurship research: Use of data envelopment analysis. Small Business Economics, 37(1), 39-57.

Apetrei, A., Sanchez, J., \& Sapena, J. (2019). The controversial link between entrepreneurial activity and inequality. International Entrepreneurship and Management Journal, 15(2), 485-502. $\begin{array}{rccc}\begin{array}{r}\text { Arenius, } \\ \text { tual }\end{array} & \text { V., \& \& } & \text { Minnitiables } \\ \text { and } & M . & \text { (2005). Percep- } & \text { Pascent } \\ \text { Entrepreneurs- }\end{array}$ hip. Small Business Economics, 24(3), 249-265. Audretsch, D., \& Fritsch, M. (2003). Linking Entrepreneurship to Growth: The Case of West of Germany. Industry and Innovation, 10(1), 65-73.

Autio, E., Keeley, R., Klofsten, M., Parker, C., \& Hay, M. (2001). Entrepreneurial intent among students in Scandinavia and in the USA. Enterprise and Innovation Management Studies, 2(2), 145-16o.

Beynon, M., Jones, P., \& Pickernell, D. (2016). Country-based comparison analysis using fsQCA investigating entrepreneurial attitudes and activity. Journal of Business Research, 69(4), 1271-1276.

Bosma, N., \& Kelley, D. (2018). Clobal Entrepreneurship Monitor: 2018/2019 Clobal Report. Retrieved abril 10, 2019, from Global Entrepreneurship Monitor: https://www.gemconsortium.org/report

Bosma, N., \& Levie, J. (2019, mayo 12). Global Entrepreneurship Monitor 2009: Clobal Report. Clobal. Retrieved from Global Entrepreneurship: http://www.gemconsortium.org/report

Cacciotti, G., Hayton, J., Mitchell, R., \& Giazitzoglu, A. (2016). A reconceptualization of fear of failure in entrepreneurship. Journal of Business Venturing, 31(3), 302-325.

Carree, M., \& Thurik, R. (2003). The Impact of Entrepre- neurship on Economic Growth. In D. Audretsch, \& Z. Acs, Handbook of Entrepreneurship Research (pp. 437-471). Boston: Kluwer-Academic Publishers.

Casson, M. (1982). The Entrepreneur: An Economic Theory. Urbana-Champaign: University of Illinois.

Castaño, M. (2012). Product innovation and R\&D policy: The case of the transformation industries in developed and developing. International Entrepreneurship and Management Journal, 8(4), 421-436.

Chang, S., \& Ahn, J. (2005). Product and Process Knowledge in the Performance-Oriented Knowledge Management Approach. Journal of Knowledge Management, 9(4), 114-132.

Charnes, A., Cooper, W., \& Rhodes, E. (1978). Measuring the efficiency of decision making units. European journal of operational research, 2(6), 429-444.

Chuang, L., Yeh, C., \& Lin, C. (2019). Applying the self-organizing maps to analyze and interpret the clustering and characteristics of nascent entrepreneurs: A cross-country study. Business \& Entrepreneurship Journal, 8(1), 29-48.

Coll, V., \& Blasco, O. (2000). Evaluación de la eficiencia mediante el análisis envolvente de datos. Valencia, España: Universidad de Valencia.

Diaz, J., Diaz, A., Sanchez, M., \& Hernandez, R. (2015). Size of government and entrepreneurship. Analysis of three groups of countries with different economic development. FAEDPYME International Review, 4(6), 45-57.

Diaz, J., Hernandez, R., Sanchez, M., \& Postigo, M. (2010). Actividad emprendedora y género. Un estudio comparativo. Revista Europea de Dirección y Economía de la Empresa, 19(2), 83-98.

Dijk, B., Den, R., Menkveld, B. , \& Thurik, R. (1997). Some New Evidence on the Determinants of Large- and Small-Firm Innovation. Small Business Economics, 9(4), 335-343.

Dilli, S., \& Westerhuis, G. (2018). How institutions and gender differences in education shape entrepreneurial activity: a cross-national perspective. Small Business Economics, 51(2), 371-392.

Elbahlawan, E. (2018). How cognitive aspects of potential entrepreneurs response to the political fluctuations and economic crisis in Egypt? Review of economics and business administration, 2(2), 289-318.

Fare, R., Grosskopf, S., Lindgren, B., \& Roos, P. (1992). Productivity changes in Swedish Pharmacies 1980-89. A nonparametric Malmquist Approach. Journal of Productivity Analysis, 13(1-2), 85-101.

Fare, R., Crosskopf, S., Norris, M., \& Zhang, Z. (1994). Productivity Growth, Technical Progress, and Efficiency Change in Industrialized Countries. The American Economic Review, 84(1), 66-83.

Farinha, L., Ferreira, J., \& Nunes, S. (2018). Linking innovation and entrepreneurship to economic growth. Competitiveness Review: An International Business Journal, 28(4), 451-475. Feeny, S., \& Rogers, M. (2003). Innovation and Per- 
formance: Benchmarking Australian Firms. The Australian Economics Review, 36(3), 253-264.

Frenkel, A., Shefer, D., Koschatzky, K., \& Walter, G. (2001). Firm Characteristics, Location and Regional Innovation: A Comparison Between Israeli and German Industrial Firms. Regional Studies, 35(5), 415-429.

Galindo, M., Mendez, M. , \&Alfaro, J.(2010). Entrepreneurship, income distribution and economic growth. International Entrepreneurship and Management Journal, 6(2), 131-141.

Global Entrepreneurship Monitor. (2019). What is GEM? Retrieved abril 12, 2019, from GEM: Clobal Entrepreneurship Monitor: https://www.gemconsortium.org/about/news

Hadjimanolis, A. (2000). A Resource-based View of Innovativeness in Small Firms. Technology Analysis \& Strategic Management, 12(2), 263-281.

Henley, A. (2007). Entrepreneurial aspiration and transition into self-employment: Evidence from British longitudinal data. Entrepreneu rship \& Regional Development, 19(3), 253-280.

Henry, C., Foss, L., Fayolle, A., Walker, E., \& Duffy, S (2015). Entrepreneurial Leadership and Gender: Exploring Theory and Practice in Global Contexts. Journal of Small Business Management, 53(3), 581-586.

Jimenez, D. \& Sanz, R. (2006). Innovación, Aprendizaje Organizativo y Resultados Empresariales. Un Estudio. Cuadernos de Economía y Dirección de la Empresa(29), 31-55.

Khan, A. (1986). Entrepreneur characteristics and the prediction of new venture success. Omega, 14(5), 365-372.

Kim, P., \& Li, M. (2014). Seeking Assurances When Taking Action: Legal Systems, Social Trust, and Starting Businesses in Emerging Economies. Organization Studies, 35(3), 359-391.

Kirzner, I. (1979). Perception, opportunity and profit: Studies in the theory of entrepreneurship. Chicago: The University of Chicago Press.

Klyver, K., \& Schenkel, M. (2013). From Resource Access to Use: Exploring the Impact of Resource Com binations on Nascent Entrepreneurship. Journal of Small Business Management, 51(4), 539-556.

Kollmann, T., Stockmann, C., \& Kensbock, J. (2017). Fear of failure as a mediator of the relationship between obstacles and nascent entrepreneurial activity-An experimental approach. Journal of Business Venturing, 32(3), 280-301.

Krueger, N. (2000). The cognitive infrastructure of opportunity emergence. Entrepreneurship: Theory and Practice, 24(3), 5-23.

Krueger, N., Reilly, M., \& Carsrud, A. (2000). Com peting models of entrepreneurial intentions. Journal of Business Venturing, 15(5-6), 411-432.

Lafuente, E., Acs, Z., Sanders, M., \& Szerb, L. (2019). The global technology frontier: Productivity growth and the relevance of Kirznerian and Schumpeterian entrepreneurship. Small Business Economics, 1-26.

Lecuna, A., Cohen, B. , \& Chavez, R. (2017). Characteristics of high-growth entrepreneurs in Latin America. International Entrepreneurshipand Management Journal,13(1), 141-159.

Leibenstein, H. (1966). Allocative Efficiency vs. "X-Efficiency". The American Economic Review, 56(3), 392-415.

Levenburg, N., Magal, S., \& Kosalge, P. (2006). An Exploratory Investigation of Organizational Factors and e-Business Motivations Among SMFOEs in the US. Electronic Markets, 16(1), 70-84

MacMillan, I., Siegel, R., \& Narasimha, S. (1985). Criteria used by venture capitalists to evaluate new venture proposals. Journal of Business Venturing, 1(1), 119-128.

McGee, J., Peterson, M., Mueller, M., \& Sequeira, J. (2009). Self-efficacy: Refining the measure. Entrepreneurship: Theory and Practice, 33(4), 965-988.

Mendez, R. (2002). Innovación y desarrollo territorial: Algunos debates teóricos recientes. Eure, XXVIII(84), 63-83.

Merino, M., \& Vargas, D. (2011). Evaluación comparativa del potencial emprendedor de Latinoamérica: Una perspectiva multinivel. Revis ta Latinoamericana de Administración(46), 38-54.

Mickiewicz, T., Wedzerai, F., Theodorakopoulos, N., \& Hart, M. (2017). Resource endowment and opportunity cost effects along the stages of entrepreneurship. Small Business Economics, 48(4), 953-976.

Noronha, M., Cesario, M., \& Fernandes, S. (2006). Interaction between Innovation in Sma-
Study. European Planning Studies, 14(1), 95-117. Park, Y., Kim, C., \& Lee, J. (1999). On The Characteristics of Innovative Firms in Korea: The Role of $R \& D$ and Innovation Type. International Journal of Innovation Management, 3(1), 111-131.

Pete, S., Nagy, A., Matis, D., Gyorfy, L., Benyovszki, A., \& Petru, T. (2011). Early-stage entrepreneurial aspirations in efficiency-driven economies. Journal for Economic Forecasting(2), 5-18.

Porter, M. (1991). La ventaja competitiva de las naciones. Barcelona: Plaza \& Janés. Qian, H., \& Acs, Z. (2013). An absorptive capacity theory of knowledge spillover entrepreneurship. Small Business Economics, 40(2), 185-197.

Ramos, A., Martinez, S., Medina, J., \& Ruiz, J. (2013). Clobal Entrepreneurship Monitor (CEM) vs. Panel Study of Entrepreneurial Dynamics (PSED): una evaluación comparativa de su estructura intelectual. Comunicación XXIII Congreso Nacional de ACEDE. Málaga.

Ruiz, J., \& Garcia, C. (2013). El fenómeno emprendedor y la convergencia Norte-Sur en Europa: Evidencias del observatorio GEM. Revista de Economía Mundial(35), 21-45.

Sanchez, Y., Garcia, F., \& Mendoza, J. (2015). La capacidad de innovación y su relación con el emprendimiento en las regiones de México. Estudios Gerenciales, 31, 243-252.

Sandberg, W., \& Hofer, C. (1987). Improving new venture performance: The role of strategy, industry structure, and the entrepreneur. Journal of Business Venturing, 2(1), 5-28.

Scheel, C. (2012). El enfoque sistémico de la innovación: Ventaja competitiva de las regiones. Estudios gerenciales, 28(o), 27-39.

Soria, K., Zuñiga, S., \& Ruiz, S. (2016). Educación e intención emprendedora en estudiantes universitarios: Un caso de estudio. Formación universitaria, 9(1), 25-34.

Stam, E. (2013). Knowledge and entrepreneurial employees: a country-level analysis. Small Business Economics, 41(4), 887-898.

Sternberg, R. (2012). Do EU Regional Policies Favour Regional Entrepreneurship? Empirical Evidence from Spain and Germany. European Planning Studies, 20(4), 583-608.

Stuetzer, M., Obschonka, M., Brixy, U., Sternberg, R., \& Cantner, U. (2014). Regional characteristics, opportunity perception and entrepreneurial activities. Small Business Economics, 42(2), 221-244.

Tasnim, N., \& Afzal, M. (2018). An empirical investigation of country level efficiency and national systems of entrepreneurship using Data Envelopment Analysis (DEA) and the TOBIT model. Journal of Global Entrepreneurship Research, 8, 1-17.

Thornhill, S. (2006). Knowledge, innovation and firm performance in high- and low-technology regimes. Journal of Business Venturing, 21(5), 687-703.

Tsai, K. Chang, H., \& Peng, C. (2016). Refining the linkage between perceived capability and entrepreneurial intention: Roles of perceived opportunity, fear of failure, and gender. International Entrepreneurship and Management Journal, 12(4), 1127-1145.

Ul Haq, M., Usman, M., Hussain, N., \& Anjam, Z. (2014). Entrepreneurial activity in China and $\mathrm{Pa}$ kistan: A GEM data evidence. Journal of Entrepreneurship in Emerging Economies, 6(2), 179-193.

Vaillant, Y., \& Lafuente, E. (2007). Do different institutional frameworks condition the influence of local fear of failure and entrepreneurial examples over entrepreneurial activity? Entrepreneurship \& Regional Development, 19(4), 313-337.

Vidal, R. (2008). Latinoamericanos: ¿Emprendedores competitivos? Revista Globalización, Competitividad y Cobernabilidad, 2(2), 126-139. Wennekers, T., \& Thurik, R. (1999). Linking Entrepreneurship and Economic Growth. Small Business Economics, 13(1), 27-55. Woodside, A., Mir, P., \& Coduras, A. (2016). The general theory of culture, entrepreneurship, innovation, and quality-of-life: Comparing nurturing versus thwarting enterprise start-ups in BRIC, Denmark, Germany, and the United States. Industrial Marketing Management, 53(1), 136-159.

Zamora, C. (2018). La importancia del emprendimiento en la economía: elcasodeEcuador. Revista Espacios, 39(7), 15-26. 